\title{
Avaliação da bioatividade de ligas de NiTi tratadas a laser para aplicação odontológica
}

\author{
Bioactivity evaluation of laser treated NiTi alloys for dental application
}

Carmem Dolores de Sá Catão*

Isabel Portela Rabello*

Anny Mirene Alves Moreira***

Marcus Vinícius Lia Fook ${ }^{* * * *}$

Carlos José de Araújo ${ }^{* * * *}$

Renata de Souza Coelho Soares ${ }^{* *}$

\section{Resumo}

As ligas de NiTi são amplamente utilizadas em dispositivos da área odontológica, como na Implantodontia, devido as suas propriedades de biocompatibilidade, memória de forma e superelasticidade. Objetivo: Avaliar a bioatividade e as características morfológicas de liga de $\mathrm{NiTi}(45,3 \%$ de Ni e $54,7 \%$ de Ti), tratadas a laser com e sem recobrimento de apatita. Materiais e método: As ligas de NiTi foram obtidas pela técnica Plasma Skull Push Pull, tratadas superficialmente. As amostras foram tratadas a laser (Yb:YAG) e pelo método biomimético em SBF-O e SBF-6 (Simulated Body Fluid), ambas a 1,5M. A análise de bioatividade foi realizada por meio da pesagem, espectroscopia por energia dispersiva de raios $X$ (EDS) e microscopia eletrônica de varredura (MEV). Resultados: As espectroscopias revelaram aumento na concentração de oxigênio e carbono após a ablação, favorecendo a difusão de átomos de oxigênio e formação de óxidos de titânio. Após a deposição da apatita pelo método biomimético em SBF-O e 6 , o recobrimento apresentou uma razão molar de $\mathrm{Ca} / \mathrm{P}$ de aproximadamente 1,0, caracterizando a formação de apatitas de baixa cristalinidade. Conclusão: $A$ associação da irradiação a laser e deposição química de apatitas pelo método biomimético em SBF-6, embora tenha demonstrado um melhor desempenho quanto à bioatividade e topografia das ligas, o ganho de massa não foi estatisticamente significativo ao nível de 5\%.
Tais propriedades podem contribuir para maior interação do biomaterial com o meio biológico e possível redução no tempo de osseointegração.

Palavras-chave: Laser. Apatitas. Implantes dentários. Osseointegração.

\section{Introdução}

Os avanços tecnológicos têm proporcionado significativos progressos na área clínica, resultando na melhoria da qualidade e aumento da expectativa de vida da população. A Odontologia tem buscado esta evolução biotecnológica para otimizar as condições psicossociais dos pacientes, utilizando-se de biomateriais, com o objetivo de substituir ou de restaurar tecidos alterados ou doentes. Dessa forma, o conhecimento dos mecanismos fisiológicos tem possibilitado o desenvolvimento e aperfeiçoamento de tecnologias na área de biomateriais ${ }^{1}$, bem como o aumento da necessidade de substituição de tecidos e órgãos de variadas complexidades em busca do biomimetismo ${ }^{2}$.

Professora Doutora, Departamento de Medicina - Universidade Federal de Campina Grande (UFCG), Campina Grande, PB, Brasil. Doutoranda em Ciências e Engenharia de Materiais - Universidade Federal de Campina Grande (UFCG), Campina Grande, PB, Brasil. Graduanda em Odontologia, Departamento de Odontologia - Universidade Federal de Campina Grande (UFCG), Patos, PB, Brasil.

Professor Doutor, Departamento de Ciências e Engenharia de Materiais - Universidade Federal de Campina Grande (UFCG), Campina Grande, PB, Brasil.

Professor Doutor, Departamento de Engenharia Mecânica - Universidade Federal de Campina Grande (UFCG), Campina Grande, PB, Brasil.

Professora Doutora, Departamento de Odontologia - Universidade Estadual da Paraíba (UEPB), Campina Grande, PB, Brasil. 
Desde então, tem-se observado uma evolução desse campo, que vai da remoção dos tecidos à sua substituição, ou ainda a regeneração tecidual, que se constitui como meta primordial a ser alcança$\mathrm{da}^{3}$. Os biomateriais são partes importantes dentro da variedade de produtos para a saúde, e devem apresentar um comportamento mecânico adequado à função que desempenham, possuir o mínimo de reação com o organismo e ser biocompatível, não estimulando ou provocando o mínimo de reações alérgicas ou inflamatórias ${ }^{4,5}$. $\mathrm{Na}$ implantodontia, sabe-se que a resistência à corrosão e degradação do implante está intimamente relacionada à boa qualidade e comportamento desse, sendo necessário um bom conhecimento das suas características e consequências do uso em longo prazo, bem como seu desempenho e resposta do hospedeiro ${ }^{6}$.

Os materiais atualmente empregados para a substituição de tecidos no corpo humano podem ser classificados segundo a sua natureza como: químicos, metálicos, plásticos, cerâmicos e compostos ${ }^{7,8}$.

A procura por materiais metálicos ou por novas ligas metálicas com propriedades satisfatórias para aplicações específicas como em implantes ortopédicos e dentários, deve-se às diferentes falhas que esses ainda apresentam, sendo tais falhas de natureza fisiológica (biocompatibilidade), mecânica (resistência) e/ou química (corrosão) ${ }^{9}$.

O NiTi é uma liga de níquel (Ni) e titânio (Ti) que apresenta propriedades de biocompatibilidade, superelasticidade e efeito memória de forma (SMA - Shape Memory Alloy), razão pela qual sua aplicação tem sido enfatizada em vários dispositivos na área médica-odontológica ${ }^{10}$. Essas propriedades podem ser modificadas por mudanças na composição da liga, trabalho mecânico e tratamentos térmicos ${ }^{11}$.

Na odontologia, o desafio é idealizar um implante dentário a partir de uma liga metálica como o $\mathrm{NiTi}$, que proporcione módulo de elasticidade próximo ao do osso, bem como resistência ao impacto e à fratura atráves da propriedade de superelasticidade da liga. Desta forma, pode-se reduzir os casos de insucessos com este tipo de tratamento, uma vez que o NiTi proporcionará outros benefícios que o titâneo isoladamente não possui ${ }^{9}$.

A rugosidade da superfície das ligas de titânio influencia as respostas teciduais e celulares, sendo, por isso, foco de inúmeras pesquisas ${ }^{13,14}$. Há diversas técnicas de modificação superficial das ligas de titânio, dentre elas, deposição de biomaterial reabsorvível como micropartículas de hidroxiapatita, plasma spray de titânio, jateamento com partículas (óxido de alumínio, silício, titânio), ataque ácido, oxidação anódica e catódica, irradiação de laser em alta intensidade ${ }^{15,16}$. Tais processos de modificação de superfície têm sido propostos com o objetivo de aumentar a área de superfície e a qualidade de adesão da interface osso-implante, baseados no princípio de que uma melhor e mais rápida osseointegração pode ser alcançada por meio da alteração topográfica do implante ${ }^{17}$. Assim, o tratamento a laser resulta na formação de um filme microporoso aderente ao substrato, que pode proporcionar uma maior interação dos tecidos biológicos com a superfície do implante, acelerando o processo de osseointegração ${ }^{15,18,19}$.

Enquanto que o recobrimento pelo método biomimético, através da imersão em SBF (Simulated Body Fluid) pode resultar na formação de uma camada de fosfato de cálcio (apatita) ${ }^{20}$, que, possivelmente, contribuirá para uma maior e mais rápida interação com o meio biológico, devido as suas propriedades como, biocompatibilidade, osteocondutibilidade, osteoindutibilidade intrínseca e, ainda, pela sua semelhança estrutural, química e física com a matriz mineral óssea ${ }^{21}$. Dessa forma, o objetivo deste trabalho foi avaliar a bioatividade e as características morfológicas de ligas de NiTi, (45,3\% de $\mathrm{Ni}$ e $54,7 \%$ de $\mathrm{Ti}$ ), tratadas a laser com e sem recobrimento de apatita.

\section{Materiais e método}

O projeto envolveu a análise de amostras de liga de NiTi $(45,3 \%$ de $\mathrm{Ni}$ e $54,7 \%$ de $\mathrm{Ti})$, previamente confeccionadas, sendo compostas pelos elementos níquel comercial puro (>99,9\%) e titânio biomédico ASTM F67-88 (grau 4).

A fabricação da liga de NiTi foi realizada no equipamento de fusão a plasma, da marca EDG, modelo Discovery All (EDG Equipamentos, São Carlos, SP, Brasil), o qual utiliza o plasma térmico como meio de transmissão de energia para fundir a maioria dos metais. Para tanto, foi empregado o processo $S k u l l$ de fusão, em que o material é fundido sobre uma fina camada dele mesmo ${ }^{22}$. O equipamento Discovery utiliza o processo de fundição e conformação através do sistema Push-Pull, no qual o molde é submetido a uma pressão de $0,2 \mathrm{MPa}(2$ $\mathrm{kg} / \mathrm{cm}^{2}$ ) na parte superior e vácuo na parte inferior, resultando em uma fundição de alta qualidade e total segurança.

As amostras foram divididas em grupos para posterior análise e comparação de resultados, de acordo com as modificações superficiais apresentadas. Os ensaios foram feitos em triplicata e as amostras classificadas em: polidas (grupo controle), com ataque superficial por laser Ytterbium pulsado, com associação de laser e deposição de apatitas por SBF-0 e, por último, ataque a laser e deposição de apatitas por SBF-6, totalizando doze amostras, divididas em três por grupo.

O procedimento de modificação superficial foi realizado no equipamento laser $Y b: Y A G$, com potência $20 \mathrm{~W}$ pulsado e frequência de $20 \mathrm{~Hz}$ (Pulsed Ytterbium Fiber laser, Sistema Omnimark 20F, Om- 
nitek Tecnologia Ltda, São Paulo, Brasil). Buscou-se caracterizar a liga de NiTi após tratamento de superfície a laser, com e sem deposição de apatita, visando aplicações biomédicas.

Parte das amostras tratadas a laser sofreram ataque químico com uma solução de hidróxido de sódio $(\mathrm{NaOH})$ com molaridade $5 \mathrm{M}$, por um período de $24 \mathrm{~h}$ a $60{ }^{\circ} \mathrm{C}$, em seguida foram lavadas em água corrente e, posteriormente, submetidas à secagem em estufa por $3 \mathrm{~h}$ a $60{ }^{\circ} \mathrm{C}$. O ataque alcalino tem em vista a ativação da superfície e a formação de titanato de sódio, que é mais reativo que o óxido de titânio, o que propicia uma deposição mais satisfatória de apatitas na superfície do biomaterial.

Dessa forma, as amostras modificadas superficialmente a laser foram submetidas ao recobrimento pelo Método Biomimético, por meio da imersão em solução SBF (Simulated Body Fluid) tipo 0 proposta por Abe et al. ${ }^{23}$ ou SBF tipo 6, estabelecida por Aparecida ${ }^{24}$, com molaridade 1,5 M. Essas soluções foram empregadas com o objetivo de analisar o efeito das modificações superficiais sobre a fase de apatita depositada de acordo com o tipo de SBF utilizado. Além disso, as SBFs foram aplicadas, por estimularem a formação de apatita, como também reduzirem o período de indução de fosfato de cálcio amorfo (ACP), proporcionando uma uniformidade da morfologia da superfície da liga após o recobrimento com apatitas ${ }^{24}$.

As amostras permaneceram imersas em SBF tipo 0 a $1 \mathrm{M}$ por um período de sete dias, mantidas em estufa a $37^{\circ} \mathrm{C} \mathrm{e} \mathrm{pH} \mathrm{7,4,} \mathrm{com} \mathrm{substituição} \mathrm{da} \mathrm{solu-}$ ção em intervalos de $24 \mathrm{~h}, 48 \mathrm{~h}$ e $48 \mathrm{~h}$, objetivando favorecer as condições de super-saturação da solução e, consequentemente, induzir a formação do recobrimento uniforme de apatita sobre a superfície da $\operatorname{amostra}^{24,25}$. Posteriormente à deposição, as amostras foram tratadas termicamente a $800{ }^{\circ} \mathrm{C}$ por uma hora, com o propósito de aumentar a cristalinidade das apatitas depositadas, através do crescimento dos cristais, densificar a camada da biocerâmica sobre o metal, bem como diminuir sua solubilidade em meio aquoso ${ }^{25,26}$, para tanto, o equipamento utilizado foi o forno da marca EDG Equipamentos, com taxa de aquecimento de $10{ }^{\circ} \mathrm{C} /$ minuto. A temperatura utilizada no tratamento térmico está de acordo com o diagrama de fases da liga, não proporcionando modificações nas condições e propriedades do substrato metálico.

Para avaliação da bioatividade, as amostras foram analisadas por Microscopia Eletrônica de Varredura (MEV) associada à Espectroscopia por Energia Dispersiva de Raios-X (EDS) TM-1000 (Hitachi High Tecnologies Corporation, Minato-ku, Tóquio, Japão). A pesagem das amostras foi realizada antes e após a imersão em SBF tipo 0 a $1 \mathrm{M}$, na balança eletrônica de precisão da marca Ohaus Adventurer, AR2140 (Ohaus Corporation, Florham Park, New Jersey, USA) com o intuito de avaliar o comporta- mento do biomaterial antes e após o contato com o fluido que mimetiza o meio biológico.

Os dados obtidos foram analisados através dos testes estatísticos T-student pareado e ANOVA, com nível de significância de 5\%, através dos programas Microsoft Excel $^{\circledR}$ versão 2010 e Statistica ${ }^{\circledR}$ versão 10.0 paraWindows, além das análises qualitativas.

\section{Resulltados}

Após as caracterizações, os resultados foram avaliados comparativamente para se estabelecer a mais adequada topografia e bioatividade nas diversas condições propostas.

Para o ensaio de bioatividade, as amostras foram pesadas antes e após a imersão em SBF-0 na concentração de $1 \mathrm{M}$, de forma a quantificar a variação em peso que cada uma delas apresentou. A partir da realização das aferições, procedeu-se a verificação da média aritmética dos ganhos, que foi tomada como parâmetro para as comparações. A Tabela 1 exibe a média dos ganhos de cada variação e evidencia que as condições superficiais que favoreceram maior ganho em peso pela deposição de apatitas em sua superfície foram as amostras tratadas a laser, seguidas das recobertas com apatita empregando-se a solução SBF-6 com molaridade de 1,5 , embora tendo sido constatadas diferenças entre as médias de ganho em massa, nas condições estabelecidas nesse estudo, essas não foram estatisticamente significante para o $\alpha$ de $5 \%$. 


\begin{tabular}{l|c}
\hline \multicolumn{1}{c|}{ Variável estudada } & Média do ganho em massa (mg) \\
\hline Liga de NiTi polidas & $0,75 \mathrm{mg}$ \\
Liga de NiTi com modificação a LASER & $1,25 \mathrm{mg}$ \\
Liga de NiTi com Método Biomimético (SBF-0) & $0,00 \mathrm{mg}$ \\
Liga de NiTi com Método Biomimético (SBF-6) & $1,00 \mathrm{mg}$ \\
\hline
\end{tabular}

As Figuras 1 (A e B), 2 (A e B), 3 (A e B) e 4 (A e B) exibem as morfologias das amostras polidas, tratadas a laser e com recobrimento por apatitas pela utilização de SBF 0 e SBF 6 (ambas a 1,5M), respectivamente, analisadas em microscópio eletrônico de varredura, antes e após a imersão em SBF tipo 0 a $1 \mathrm{M}$ para o ensaio de bioatividade. Para melhor associação e entendimento do comportamento do biomaterial quando em contato com meio biológico, os resultados do EDS encontram-se seguidos das micrografias, correspondendo às Figuras $1(\mathrm{C}$ e D), 2 (C e D), 3 (C e D) e 4 (C e D).
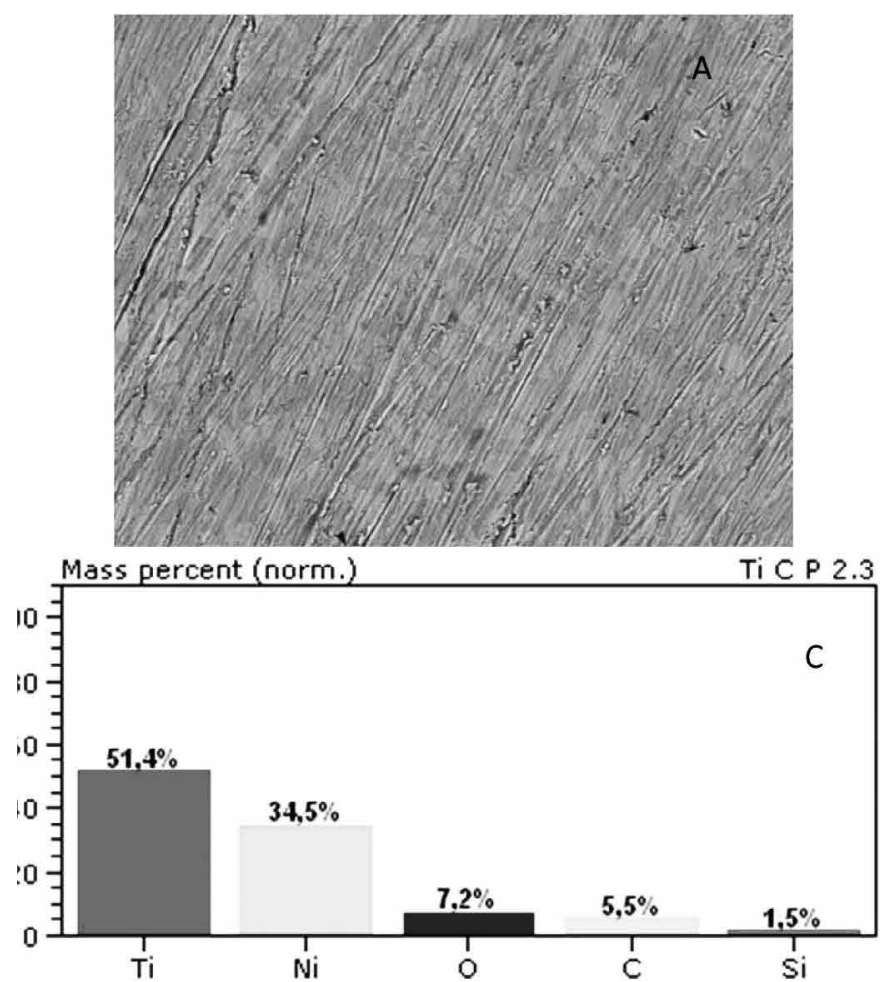

A Figura 1 (A) exibe, no primeiro momento, as superfícies da liga polida, com presença de riscas originárias do processo de corte e polimento. Após a imersão em SBF-0 para o ensaio da bioatividade, pode-se perceber a deposição de cristais superficiais (Fig. 1 B), que foram identificados com o auxílio da análise por EDS (Fig. $1 \mathrm{C}$ ).

Figura 1 - MEV de liga de NiTi polida com aumento de 2000x antes (A) e após (B) a imersão em SBF-0, e elementos químicos presentes nas amostras analisadas por EDS antes (D) e após (E) a imersão em SBF tipo 0 para o ensaio de bioatividade

A Figura 2 (A e B) evidencia a topografia da liga de NiTi modificada por laser e analisada em MEV, antes e após a imersão em SBF tipo 0. A Figura 2 $(\mathrm{C}$ e D) representa os resultados do EDS correspondentes às imagens. 

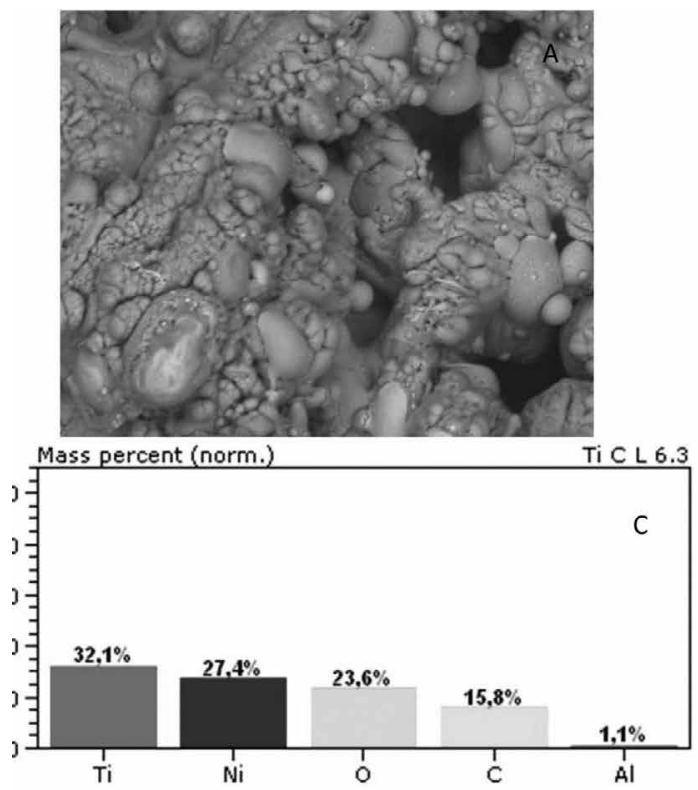

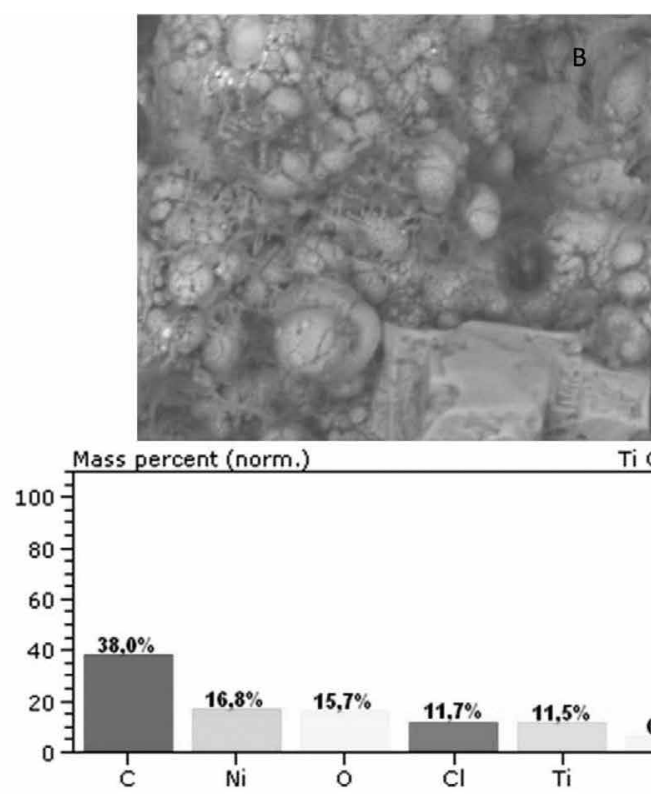

Figura 2 - MEV da liga de NiTi modificada por laser com aumento de 2000x antes (A) e após (B) a imersão em SBF-0, e elementos químicos presentes nas amostras analisadas por EDS antes $(C)$ e após $(D)$ a imersão em SBF tipo 0 para o ensaio de bioatividade.

De acordo com a análise da Figura 2 (A e B), observa-se a presença de estruturas circulares em toda superfície das amostras que, analisadas em EDS, apontam a formação de cristais de cloreto de sódio e a existência de outros compostos, como: Titânio (Ti), Níquel (Ni), Carbono (C) e Oxigênio (O). O Alumínio (Al) e o Silício (Si) existentes na análise podem ser considerados como agentes contaminantes, em virtude da baixa concentração (Fig. 2 C e D).

No presente estudo foi empregada a solução SBF0 preconizada por Abe et al. ${ }^{23}$ (1990) e a solução SBF-6 proposta por Aparecida ${ }^{24}$ (2006), ambas a 1,5M, a fim de conciliar as propriedades mecânicas da liga que se aproximam às condições encontradas no osso
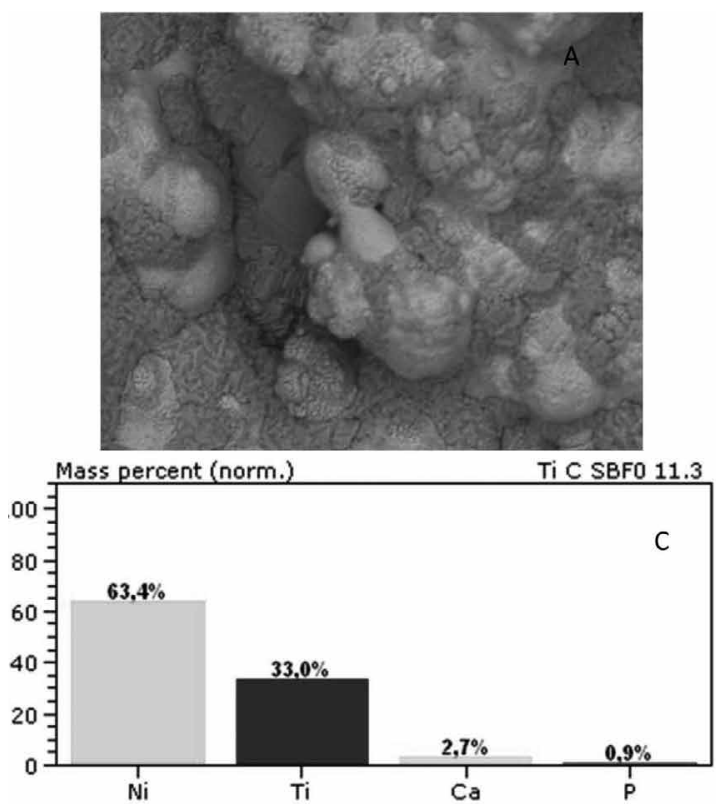

humano com a bioatividade, reduzindo o tempo de recobrimento e propiciando a formação de compostos de apatitas de interesse biológico.

As micrografias representadas na Figura 3 (A e B) apresentam a microestrutura da camada de apatita obtida pelo Método Biomimético empregando-se as soluções SBF tipo 0 e 6 , respectivamente. As análises foram realizadas antes e após a imersão em SBF tipo 0 para o ensaio de bioatividade. A morfologia das ligas modificadas por irradiação a laser apresentou nucleações homogêneas, com esferas e tamanhos de partículas em escala micrométrica (utilizando-se como referência a escala do microscópio), superfícies rugosas, com presença de macro e micro poros.
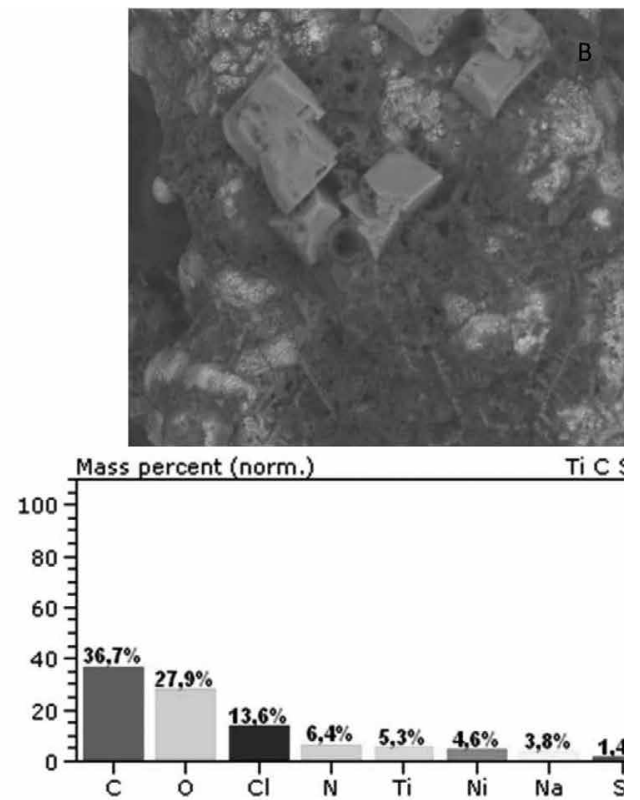

Figura 3 - MEV da liga NiTi modificada por laser e com deposição de apatitas por SBF-0 com aumento de 2000x antes (A) e após (B) a imersão em SBF-0, e elementos químicos nas amostras analisadas por EDS antes (C) e após (D) a imersão em SBF tipo 0 para o ensaio de bioatividade. 
As Figuras 3 (C e D) e 4 (C e D) exibem os resultados do EDS correspondentes à modificação pelo Método Biomimético com SBF-0 e SBF-6, res-

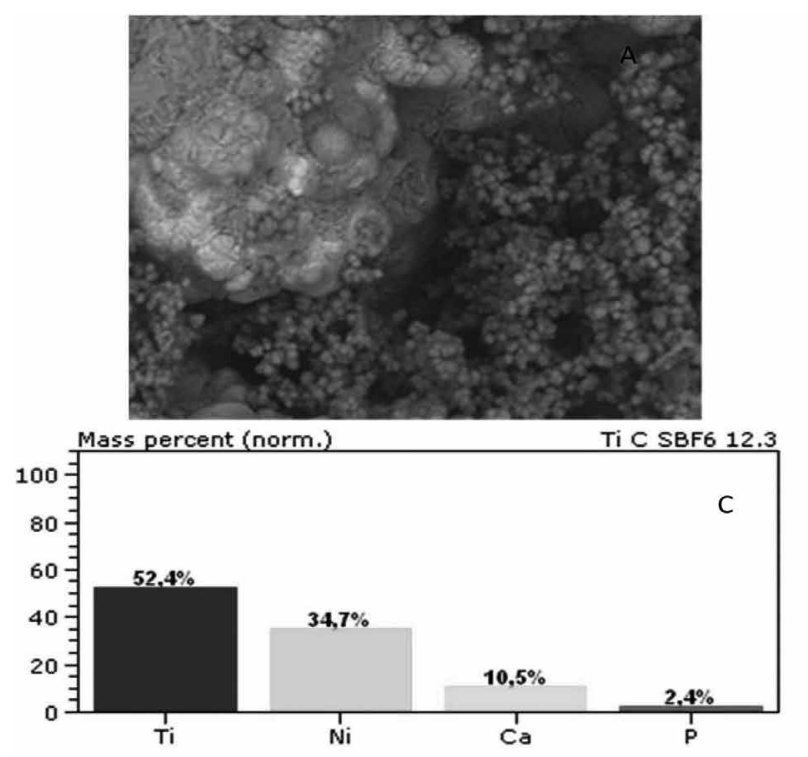

pectivamente, onde percebe-se um acréscimo do percentual em massa de compostos presentes nas soluções utilizadas.

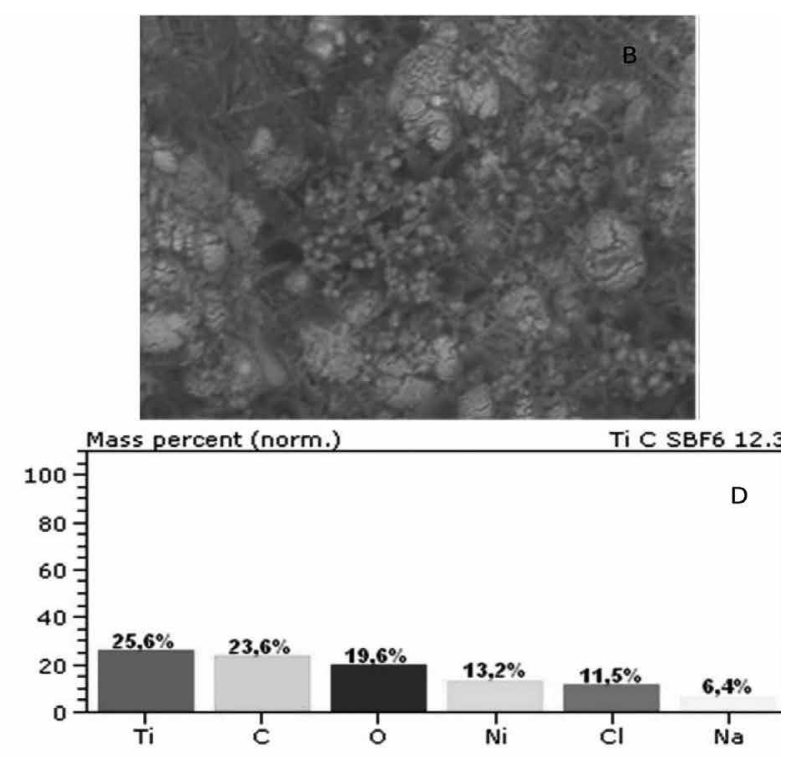

Figura 4 - MEV de liga NiTi modificada por laser e com deposição de apatitas por SBF-6 com aumento de 2000x antes (A) e após (B) a imersão em SBF-O, e elementos químicos presentes nas amostras analisadas por EDS antes (C) e após (D) a imersão em SBF tipo 0 para o ensaio de bioatividade.

\section{Discussão}

Com relação às amostras que foram apenas polidas, verificou-se que o polimento proporcionou uniformidade superficial, porém com presença de linhas unidirecionais decorrentes das etapas de lixamento e polimento.

A análise topográfica das amostras submetidas ao tratamento a laser revelou a presença de diferentes camadas de óxidos, por meio da mudança no tempo de exposição da superfície ao feix ${ }^{27}$. Além disso, a acentuação da rugosidade na superfície resultou da rápida fusão e solidificação durante o processo de ablação a laser, e as irregularidades uniformemente distribuídas pela superfície do biomaterial podem proporcionar maior interação com as células do meio biológico, resultando em uma precoce osseointegração $0^{15,18}$.

A ablação a laser proporcionou a formação de aglomerados circulares em toda superfície das amostras, intercalados por fendas relativamente regulares, exibindo porosidade, rugosidade e morfologia mais homogênea, estando de acordo com os trabalhos de $\operatorname{Sisti}^{28}$ (2011) e Tavares ${ }^{29}$ (2009) em que se encontram topografias semelhantes às obtidas neste estudo.

O fenômeno de ablação associa-se à ruptura em escala macroscópica da superfície devido ao processo de fusão e solidificação rápido na superfície do metal, o que conduz à perda da estequiometria, gerando um estado de menor estabilidade e alterações de composição da superfície do material ${ }^{15}$. Pode-se observar que o processo de ablação proporcionou aumento na rugosidade da superfície atacada em todas as amostras, de forma a proporcionar aumento da área superficial, característica desejada para osseointegração, uma vez que as respostas celulares (migração, ancoragem, diferenciação e expressão da matriz) são afetadas pelas características topográficas e pela composição química da superfície ${ }^{30}$.

Observou-se que as amostras modificadas superficialmente por laser e que entraram em contato com SBF pelo Método Biomimético apresentaram deposição de apatitas, caracterizada pela presença de grãos esféricos nas superfícies das amostras submetidas a ambas soluções, havendo diferenças topográficas significativas entre as composições de NiTi imersas em SBF-0, proposto por Abe et al. ${ }^{23}$ (1990) e SBF-6, estabelecido por Aparecida $^{24}$ (2006), havendo nesta última a formação de uma camada mais espessa de apatitas. Em contrapartida, nas amostras que entraram em contato com SBF-0 a 1,5M pode-se observar a presença de cristais alongados que possivelmente representam uma variação de apatita, corroborando os resultados encontrados por Catão ${ }^{9}$. Os resultados revelaram que a modificação da superfície por laser favorece a formação de grupos de apatitas, mesmo em diferentes concentrações de SBF.

Ao analisar as imagens obtidas pelo MEV, pode-se perceber que as amostras modificadas superficialmente por laser e deposição de apatitas por SBF tipo 6 apresentaram quantidade significativamente 
maior de apatitas em sua superfície do que as amostras cuja deposição foi a partir de SBF-0. Esse resultado reitera o estudo de Aparecida ${ }^{24}$ quanto à indicação de SBF-6 para fins de deposição superficial, visto que as diferenças entre as deposições proporcionadas - SBF tipo 0 e tipo 6 - foram consideráveis.

A estreita variação de deposição de apatita existente entre as amostras imersas em SBF 0 e 6 pode estar relacionada à composição das soluções. Mesmo empregando-se soluções SBF que favorecem a deposição de apatitas de interesse biológico (Fosfato tricálcico - TCP, Fosfato octacálcico - OCP e Hidroxiapatita - HA), não se pode especificar precisamente o tipo de apatita depositada nas condições empregadas nessas amostras. Contudo, pode-se afirmar a existência da formação de um filme biocerâmico, fato avaliado pela uniformidade das morfologias apresentadas nas microscopias, sobretudo nas amostras imersas em SBF 6.

A formação de uma camada de apatita biologicamente ativa na interface material-osso é um dos métodos capazes de tornar a superfície de um material bioativa. Essa apatita é semelhante, em composição e estrutura, à fase mineralizada do tecido ósseo e atua atraindo células e proteínas para iniciar as reações que resultam na formação da estrutura óssea ${ }^{31}$.

Assim, ao avaliar a bioatividade em liga de NiTi, percebeu-se que a pesagem das amostras corrobora as características topográficas, nas quais após o tratamento de superfície a laser, ocorreu um aumento da rugosidade superficial, formação de uma superfície porosa e homogênea, com defeitos lineares maiores e mais profundos, o que proporcionou um aumento da superfície de contato e, possivelmente, a presença de uma mistura de óxidos estequiométricos e não estequiométricos com diferentes morfologias e propriedades ${ }^{28}$, características morfológicas que, possivelmente, favoreceram a deposição de apatita.

$\mathrm{O}$ estudo de recobrimento por apatita, empregando-se o Método Biomimético em superfície de titânio, revelou que na solução SBF-0 a presença de elementos Ca e P numa razão de 1,45, evidenciou uma mistura de fases correspondente ao fosfato octacálcico (OCP) e hidroxiapatita deficiente de cálcio. Já na solução $\mathrm{SBF}-6$, a razão $\mathrm{Ca} / \mathrm{P}$ foi de 1,53 , o que demonstra que a solução SBF-6 tende a favorecer a deposição de apatita de interesse biológico ${ }^{24}$.

O ensaio de EDS, após a utilização do Método Biomimético em SBF-0 e SBF-6, foi empregado para verificar composição química elementar, como a presença de Fósforo (P) e Cálcio (Ca), na tentativa de identificar o tipo de fosfato de cálcio presente na superfície da amostra através do cálculo da relação $\mathrm{Ca} / \mathrm{P}$. As amostras apresentaram-se com uma razão molar de aproximadamente 1,0, representando um valor médio da relação $\mathrm{Ca} / \mathrm{P}$. Sendo assim, pode-se afirmar que, possivelmente, estejam presentes outros fosfatos de cálcio além da hidroxiapatita. No segundo momento, após a imersão em SBF-0 para o ensaio da bioatividade, foram encontrados os elementos C, Ti, Ni, Cl, Na, P, Ca, O, Si e Mg (magnésio). Nesse segundo ensaio, não houve interferência para identificação de um elemento químico em especial, já que a intenção era a identificação de todos os componentes presentes na superfície.

A interação dos elementos C (carbono) e O (oxigênio), presentes na amostra, podem resultar na formação de carbeto de titânio (TiC) e óxido de titânio $\left(\mathrm{TiO}_{2}\right)$. A camada passiva de óxido de titânio forma-se rapidamente quando o metal entra em contato com oxigênio, sendo um dos óxidos conhecidos mais estáveis.

Dessa forma, o tratamento superficial a laser ou a combinação com a deposição de apatita pelo Método Biomimético em ligas de NiTi promoveu uma microtopografia que pode otimizar a interação com as células em meio biológico, reduzindo o tempo de osseointegração. A textura superficial de um biomaterial, incluindo a microtopografia e rugosidade, tem sido relatada como um fator que pode influenciar no sucesso da resposta dos implantes osseointegráveis ${ }^{32}$.

\section{Conclusão}

As análises laboratoriais evidenciaram que as amostras de NiTi modificadas por laser, associadas ou não à apatita, apresentaram superfícies com propriedades morfológicas que podem contribuir para uma precoce osseointegração, por possuírem maior rugosidade e área de contato, além da presença de apatitas, que promovem mais rápida adesão do biomaterial ao tecido ósseo, sendo, portanto, mais adequadas para a utilização como biomaterial. Conclui-se que, nas condições estabelecidas para síntese e modificações superficiais da liga de NiTi, o tratamento superficial que obteve o melhor desempenho foi o que associou a irradiação a $l a-$ ser com a deposição química de apatitas através do Método Biomimético empregando a solução SBF-6, embora um dos requisitos para avaliação da bioatividade, como ganho em massa, não tenha sido estatisticamente significante.

\section{Abstract}

NiTi alloys are extensively used in devices for dentistry, as in Implantology, due to their biocompatibility properties, shape memory, and superelasticity. Objective: To assess bioactivity and morphological characteristics of NiTi alloy (45.3\% Ni and $54.7 \% \mathrm{Ti})$, treated with laser and uncoated apatite. Materials and method: The NiTi alloys were obtained by the Plasma Skull Push-Pull process, superficially treated. The samples were treated with laser (Yb: YAG), and by the biomimetic method in SBF-O and SBF-6 (Simulated Body Fluid), both with 1.5M. Bioactivity analysis was performed by weighing, 
Energy Dispersive X-Ray Spectroscopy (EDS), and Scanning Electron Microscopy (SEM). Results: Spectroscopies revealed an increase in the concentration of oxygen and carbon after ablation, favoring the dissemination of oxygen atoms, and the formation of titanium oxides. After the deposition of apatite by the biomimetic method in $S B F-O$ and 6, the coating showed a molar ratio of $\mathrm{Ca} / \mathrm{P}$ of approximately 1.0, characterizing the formation of poor crystallinity apatites. Conclusion: The combination of laser irradiation and chemical deposition of apatites by the biomimetic method in SBF-6, although performing better in terms of bioactivity and topography of alloys, the mass gain was not statistically significant at the 5\% level. Such properties may contribute to greater interaction of the biomaterial with biological environment and possible time reduction for osseointegration.

Keywords: Laser. Apatites. Dental Implants. Osseointegration.

\section{Referências}

1. Tomás HMPG. Avaliação do comportamento à corrosão e da citocompatibilidade de uma liga ortopédica de Co-Cr-Mo [Tese de Doutorado]. Lisboa: Universidade de Lisboa FCUL; 1995.

2. John KRS, Zardiackas LD, Black RJ, Armstrong R. Response of canine bone to a synthetic bone graft material. Clin Mater 1993; 12:49-55.

3. Hench LL. Biomaterials: a forecast for the future. Biomaterials $1998 ; 19: 1419-23$.

4. Souza-Filho BS, Araújo CJ, Guastaldi AC, Carvalho LH. Caracterização de materiais com efeito memória de forma a base de Ni-Ti tratadas a laser para aplicação médica. Ver Eletr Mater Proces. 2011; 6(1):59-76.

5. Soares GAS. Biomateriais: classificação, seleção e avaliação. Rio de Janeiro: COPPE/UFRJ; 2002.

6. Williams DF, Williams RL. Degradative effects of the biological environmental metals and ceramics. In: Ratner BD, Hoffman AS, Achoen FJ, Lemons JE. Biomaterials Science an introduction to materials in medicine. San Diego: Academic Press; 1996. p. 260-67.

7. Mears DC. Metals in medicine and surgery. Intern Metals Rev 1997; 218:119-55.

8. Disegi JA, Eschback L. Stainless steel in bone surgery. Injury $2000 ; 31: 2-6$.

9. Catão CDS. Obtenção e Avaliação de Ligas de Nitinol tratadas a LASER: Perspectiva para Aplicação em Implantes Dentários [Tese de Doutorado]. Campina Grande: Universidade Federal de Campina Grande; 2011.

10. Krone L, Mentz J, Bram M, Buchkremer HP, Stover D. The potential of powder metalurgy for the fabrication of biomaterials on the basis of Nickel-Titanium: A case study with a staple showing shape memory behaviour. Advan Engin Mater 2005; 7:613-9.

11. Mertmann M. Memory-Metalle, Weil amRhein [homepage na internet]. Germany:Technology \& Services; 2000 [atualizada em 2000 Aug 27; acesso em 2011 Aug 18]. NiTi: the material for implantsandinstruments; [aproximadamente 2 telas]. Disponível em: http://www.memorymetalle.com.de
12. Puleo DA, Nanci A. Understanding and controlling the bone-implant interface. Biomaterials. 1999; 20(23):2311-21.

13. Cooper LF. A role for surface topography in creating and maintaining bone at titanium endosseous implants. J Prosth Dentistry 2000; 84:522-34.

14. Le Gue'Hennec L, Soueidan A, Layrolle P, Amouriq Y. Surface treatments of titanium dental implants for rapid osseointegration. Dent Mater 2007; 23:844-54.

15. György E, Pérez Del Pino A, Serra P, Morenza JL. Chemical composition of dome-shaped structures grown on titanium by multi-pulse Nd:YAG LASER irradiation. Appl Surface Science 2004; 222:415-22.

16. Sisti KE, Garcia Júnior IR, Guastaldi AC, Antoniolli ACMB, De Rossi R, Brochado Neto AL. Analyse titanium surface irradiated with LASER, with and without deposited of durapatite. Acta Cir Bras 2006; 21(4):57-62.

17. Klokkevold PR, Nishimura RD, Adachi M, Caputo A. Osseointegration enhanced by chemical etching of the titanium surface: a torque removal study in the rabbit. Clin Oral Implants Research 1997; 8(6):442-7.

18. De Carvalho BM, Pellizzer EP, De Moraes SLD, Falcón-Antenucci R, Ferreira Júnior JS. Tratamentos de superfície nos implantes dentários. Rev Cir Traumatol Buco Maxilofac 2009; 9(1):123-30.

19. Lavisse L, Grevey D, Langlade C, Vannes B. The early stage of the LASER-induced oxidation of titanium substrates. Appl Surface Science 2002; 186:150-5.

20. Godley R, Starosvetsky D, Gotman I. Bonelike apatite formation niobium metal treated in aqueous $\mathrm{NaOH}$. J Mater Science: Mater in Med 2004; 15(10):1073-7.

21. Storz O, Gasthuber H, Woydt M. Tribological properties of thermalsprayed Magnéli-type coatings with different stoichiometries $\left(\mathrm{TinO}_{2} \mathrm{n}-1\right)$. Surface \& Coatings Technol 2001; 140:76-81.

22. EDG Equipamentos e Controles Ltda. DISCOVERY Plasma: Manual de instalação, operação e manutenção. São Paulo; 2005.

23. Abe Y, Kokubo T, Yamamuro T. Apatite coating on ceramics, metals and polymers utilizing a biological process. J Mater Science: Mater in med 1990; 1(4):233-8.

24. Aparecida AH. Recobrimento de apatitas empregando-se o método biomimético: Estudo da Influência dos Íons $\mathrm{K}^{+}, \mathrm{Mg}^{2+}$, $\mathrm{SO}_{4}{ }^{2-}$ e $\mathrm{HCO}_{3}$ na formação de hidroxiapatita [Dissertação de Mestrado]. Araraquara: Instituto de Química da Universidade Estadual Paulista; 2006.

25. Fook MVL. Desenvolvimento da técnica de deposição de hidroxiapatita na superfície de polietileno de ultra-peso molecular para aplicação como biomaterial [Tese de Doutorado]. Araraquara: Instituto de Química da Universidade Estadual Paulista; 2005.

26. Almeida Filho E. Avaliação do Tratamento Térmico em Recobrimentos Cerâmicos Bioativos pelo Método Biomimético sobre Titânio c.p. modificados pelo Laser Nd:YAG [Dissertação de Mestrado]. Araraquara: Instituto de Química da Universidade Estadual Paulista; 2008.

27. Hsu SH, Liu BS, Lin WH, Chiang HC, Huang SC. Characterization and biocompatibility of a titanium dental implant with a laser irradiated and dual-acid et chad surface. Biomed Mater Eng 2007; 17:53-68. 
28. Sisti EK. Estudo da topografia e da osseointegração de implante de titânio sob ação do laser, com ou sem deposição de hidroxiapatita em coelhos [Tese de Doutorado]. Campo Grande: Universidade Federal de Mato Grosso do Sul; 2011.

29. Tavares HS. Estudo in vitro da interação de titânio irradiado por feixe de laser Yb:YAG com e sem recobrimento de apatitas, empregando-se cultura de células estaminais humana [Tese de Doutorado]. Araraquara: Instituto de Química da Universidade Estadual Paulista; 2009.

30. Guastaldi AC. Engenharia de superfície do titânio para aplicações biomédicas. Metalurgia e Materiais 2003; 59(535):442-4.

31. Kokubo T, Kim HM, Nishiguchi S, Nakamura T. In vivo apatite formation induced on titanium metal and its alloys by chemical treatment. Key Engineering Materials. 2001; 192-195:3-6.

32. Chen MF, Yang XJ, Hu RX, Cui ZD, Man HC. Bioactive NiTi shape memory alloy used as bone bonding implants. Materials Science and Engineering 2004; 6(1):497-502.

\section{Endereço para correspondência:}

Carmem Dolores de Sá Catão

Universidade Federal de Campina Grande

Unidade Acadêmica de Ciências e da Saúde

Juvêncio Arruda, 795, Bodocongó

58407-485 Campina Grande/PB

Fones: +55 83 2101-1239 / +5583 8883-1110

E-mail: cataocarmem@gmail.com

Recebido: 10/11/2013. Aceito: 03/04/2014. 